\title{
Structural insights into interaction mechanisms of alternative piperazine-urea YEATS domain binders in MLLT1
}

Xiaomin $\mathrm{Ni}^{1,2}$, David Heidenreich ${ }^{1,2}$, Thomas Christott ${ }^{3}$, James Bennett ${ }^{3}$, Moses Moustakim ${ }^{3, \dagger}$, Paul E. Brennan, Oleg Fedorov ${ }^{3}$, Stefan Knapp ${ }^{1,2 *}$, Apirat Chaikuad ${ }^{1,2 *}$

${ }^{1}$ Institute of Pharmaceutical Chemistry, Goethe-University Frankfurt, 60438 Frankfurt, Germany

${ }^{2}$ Structural Genomics Consortium, BMLS, Goethe-University Frankfurt, 60438 Frankfurt, Germany

${ }^{3}$ Target Discovery Institute and Structural Genomics Consortium, University of Oxford, Oxford OX3 7DQ, UK

Supplementary Information

Supplementary table S1 Data collection and refinement statistics

Supplementary table S1 Data collection and refinement statistics

2

Supplementary table S2 Summary of IC50s of the compounds against other YEATS domains. 4

Supplementary figure S1 Omitted electron density maps for the bound ligands 4 
Supplementary table S1 Data collection and refinement statistics

\begin{tabular}{|c|c|c|c|}
\hline Complex & MLLT1-1 & MLLT1-2 & MLLT1-3 \\
\hline PDB accession code & 6T1I & 6T1J & 6T1L \\
\hline \multicolumn{4}{|l|}{ Data Collection } \\
\hline Resolutiona $^{\mathrm{a}}(\AA)$ & $48.94-1.80(1.86-1.80)$ & $49.15-1.97(2.04-1.97)$ & $49.08-2.00(2.07-2.00)$ \\
\hline Spacegroup & $P 4_{3} 2_{1} 2$ & $P 4_{3} 2_{1} 2$ & $P 4_{3} 2_{1} 2$ \\
\hline Cell dimensions & $\begin{array}{l}a=\mathrm{b}=48.9, c=132.8 \AA \\
\alpha, \beta, \gamma=90.0^{\circ}\end{array}$ & $\begin{array}{l}a=\mathrm{b}=49.2, c=131.0 \AA \\
\alpha, \beta, \gamma=90.0^{\circ}\end{array}$ & $\begin{array}{l}a=\mathrm{b}=49.1, c=131.7 \AA \\
\alpha, \beta, \gamma=90.0^{\circ}\end{array}$ \\
\hline No. unique reflections $\mathrm{s}^{\mathrm{a}}$ & $15,758(1,499)$ & $12,088(1,139)$ & $11,625(1,105)$ \\
\hline Completeness $^{\mathrm{a}}(\%)$ & $100.0(100.0)$ & $100.0(100.0)$ & $100.0(100.0)$ \\
\hline $\mathrm{I} / \sigma \mathrm{I}^{\mathrm{a}}$ & $13.7(2.2)$ & $9.0(2.1)$ & $12.3(2.2)$ \\
\hline $\mathrm{R}_{\text {merge }}{ }^{\mathrm{a}}(\%)$ & $0.071(0.806)$ & $0.104(0.768)$ & $0.086(0.889)$ \\
\hline $\mathrm{CC}(1 / 2)$ & $0.999(0.891)$ & $0.997(0.917)$ & $0.998(0.916)$ \\
\hline Redundancy ${ }^{\mathrm{a}}$ & $9.6(9.9)$ & $9.5(8.8)$ & $9.9(10.3)$ \\
\hline \multicolumn{4}{|l|}{ Refinement } \\
\hline No. atoms in refinement $(\mathrm{P} / \mathrm{L} / \mathrm{O})^{\mathrm{b}}$ & $1,182 / 27 / 98$ & $1,182 / 29 / 74$ & $1,177 / 28 / 81$ \\
\hline $\mathrm{B}$ factor $(\mathrm{P} / \mathrm{L} / \mathrm{O})^{\mathrm{b}}\left(\AA^{2}\right)$ & $44 / 50 / 51$ & $53 / 83 / 58$ & $55 / 88 / 60$ \\
\hline $\mathrm{R}_{\text {fact }}(\%)$ & 20.5 & 22.4 & 21.8 \\
\hline $\mathrm{R}_{\text {free }}(\%)$ & 23.2 & 27.5 & 26.5 \\
\hline rms deviation bond ${ }^{\mathrm{c}}(\AA)$ & 0.013 & 0.011 & 0.010 \\
\hline rms deviation angle $\left(^{\mathrm{c}}\right)$ & 1.3 & 1.2 & 1.2 \\
\hline \multicolumn{4}{|l|}{ Molprobity Ramachandran } \\
\hline Favour $(\%)$ & 97.16 & 93.62 & 95.00 \\
\hline Outlier (\%) & 0 & 0 & 0 \\
\hline
\end{tabular}

${ }^{a}$ Values in brackets show the statistics for the highest resolution shells.

${ }^{\mathrm{b}} \mathrm{P} / \mathrm{L} / \mathrm{O}$ indicate protein, ligand molecules, and other (water and solvent molecules), respectively.

${ }^{\mathrm{c}} \mathrm{rms}$ indicates root-mean-square. 
Supplementary table S1 Data collection and refinement statistics (continued)

\begin{tabular}{|c|c|c|c|}
\hline Complex & MLLT1-4 & MLLT1-5 & MLLT1-6 \\
\hline PDB accession code & 6T1M & 6T1N & 6T10 \\
\hline \multicolumn{4}{|l|}{ Data Collection } \\
\hline $\operatorname{Resolution}^{\mathrm{a}}(\AA)$ & $49.07-1.85(1.91-1.85)$ & $49.01-1.95(2.02-1.95)$ & $49.03-1.90(1.97-1.90)$ \\
\hline Spacegroup & $P 4{ }_{3} 2{ }_{1}$ & $P 4{ }_{3} 2{ }_{1}$ & $P 4_{3} 2_{1} 2$ \\
\hline Cell dimensions & $\begin{array}{l}a=\mathrm{b}=49.1, c=130.0 \AA \\
\alpha, \beta, \gamma=90.0^{\circ}\end{array}$ & $\begin{array}{l}a=\mathrm{b}=49.0, c=132.4 \AA \\
\alpha, \beta, \gamma=90.0^{\circ}\end{array}$ & $\begin{array}{l}a=b=49.0, c=131.4 \AA \\
\alpha, \beta, \gamma=90.0^{\circ}\end{array}$ \\
\hline No. unique reflections $\mathrm{s}^{\mathrm{a}}$ & $14,360(1,368)$ & $12,512(1,201)$ & $13,008(1,212)$ \\
\hline Completeness $^{\mathrm{a}}(\%)$ & $100.0(100.0)$ & $100.0(100.0)$ & $97.5(94.8)$ \\
\hline $\mathrm{I} / \sigma \mathrm{I}^{\mathrm{a}}$ & $10.6(2.1)$ & $9.8(2.2)$ & $8.7(2.6)$ \\
\hline $\mathrm{R}_{\text {merge }}{ }^{\mathrm{a}}(\%)$ & $0.087(0.800)$ & $0.082(0.735)$ & $0.077(0.418)$ \\
\hline $\mathrm{CC}(1 / 2)$ & $0.998(0.930)$ & $0.997(0.830)$ & $0.991(0.913)$ \\
\hline Redundancy $^{\mathrm{a}}$ & $10.0(10.4)$ & $7.1(7.4)$ & $4.8(4.5)$ \\
\hline \multicolumn{4}{|l|}{ Refinement } \\
\hline No. atoms in refinement $(\mathrm{P} / \mathrm{L} / \mathrm{O})^{\mathrm{b}}$ & $1,185 / 27 / 76$ & $1,172 / 26 / 99$ & $1,178 / 107^{d} / 51$ \\
\hline $\mathrm{B}$ factor $(\mathrm{P} / \mathrm{L} / \mathrm{O})^{\mathrm{b}}\left(\AA^{2}\right)$ & $50 / 58 / 56$ & $42 / 46 / 50$ & $38 / 43 / 47$ \\
\hline $\mathrm{R}_{\text {fact }}(\%)$ & 21.5 & 20.9 & 20.2 \\
\hline $\mathrm{R}_{\text {free }}(\%)$ & 27.5 & 26.6 & 25.1 \\
\hline rms deviation bond $(\AA)$ & 0.013 & 0.010 & 0.013 \\
\hline rms deviation angle ${ }^{\mathrm{c}}\left({ }^{\circ}\right)$ & 1.3 & 1.2 & 1.3 \\
\hline \multicolumn{4}{|l|}{ Molprobity Ramachandran } \\
\hline Favour (\%) & 97.86 & 98.56 & 97.12 \\
\hline Outlier (\%) & 0 & 0 & 0 \\
\hline
\end{tabular}

a Values in brackets show the statistics for the highest resolution shells.

${ }^{b} \mathrm{P} / \mathrm{L} / \mathrm{O}$ indicate protein, ligand molecules, and other (water and solvent molecules), respectively.

${ }^{\mathrm{c}}$ rms indicates root-mean-square.

d ligand modelled in two conformations. 
Supplementary table S2 Summary of IC50s of the compounds against other YEATS domains.

\begin{tabular}{|l|c|c|c|}
\hline & \multicolumn{2}{|l|}{ IC50 $(\boldsymbol{\mu M})$} \\
\hline $\mathbf{c o m p o u n d}$ & MLLT3 & YEATS2 & YEATS4 \\
\hline $\mathbf{1}$ & 7.8 & 76.8 & $>100$ \\
\hline $\mathbf{3}$ & 33.0 & 32.2 & $>100$ \\
\hline $\mathbf{4}$ & $>100$ & $>100$ & $>100$ \\
\hline $\mathbf{5}$ & n.d & $>100$ & $>100$ \\
\hline $\mathbf{6}$ & n.d & 53.2 & $>100$ \\
\hline
\end{tabular}

Supplementary figure S1 Omitted electron density maps for the bound ligands. The $\left|F_{O}\right|-\left|F_{C}\right|$ electron density maps contoured at $2.5 \sigma$.

1

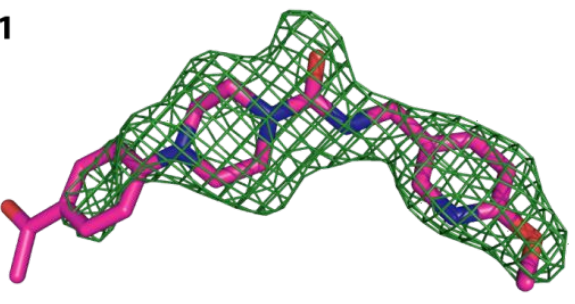

2

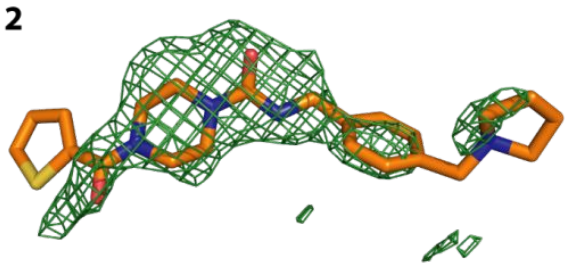

3

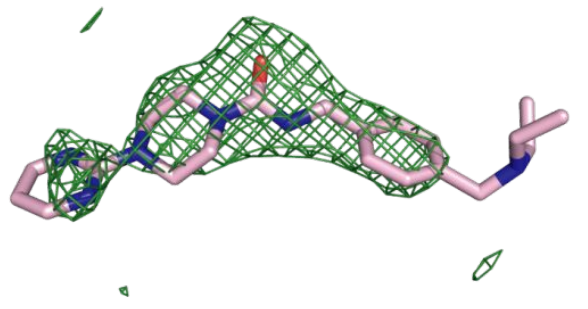

4

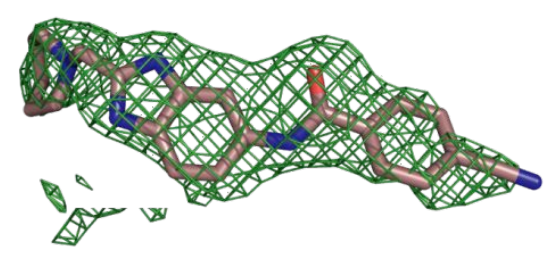

5

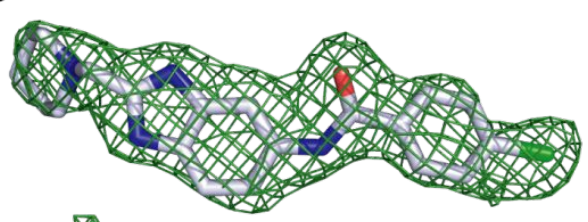

$\bigotimes$

$v-$

6

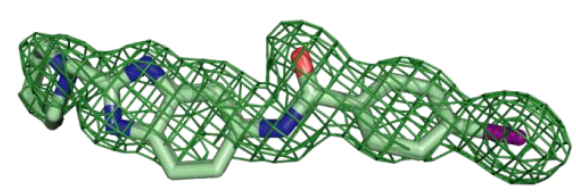

